\section{Low QRS voltage and atrial fibrillation precluding implan- tation of a subcutaneous implantable cardioverter- defibrillator in a patient with arrhythmogenic cardiomyopathy}

Peter C. Kahr, ${ }^{1,2}$ Jan Steffel, ${ }^{1}$

Alexander Breitenstein, ${ }^{1}$

Thomas Wolber, ${ }^{1}$ Laurent M. Haegeli,

Deniz Akdis, ${ }^{1}$ Firat Duru, ${ }^{1}$

Corinna Brunckhorst, ${ }^{1}$

Ardan M. Saguner ${ }^{1}$

${ }^{1}$ Department of Cardiology, University

Heart Centre Zurich; ${ }^{2}$ Department of

Medicine, University Hospital Zurich,

Zurich, Switzerland

\begin{abstract}
Arrhythmogenic cardiomyopathy (AC) is a rare mostly hereditary disease, in which fibro-fatty tissue replaces cardiomyocytes. Typically, the first alterations of the disease can be encountered in the epicardium of the right ventricle in adolescent patients. From there, the disease usually progresses over time. Besides the development of heart failure, the clinical significance of the disease is determined by the predisposition to potentially lethal ventricular arrhythmias. Hence, a majority of patients with AC require an implantable cardioverter-defibrillator (ICD) to be protected from sudden cardiac death. A recently developed alternative to transvenous systems are subcutaneous ICDs (S-ICD), associated with a lower risk of device-related complications such as endocarditis since no foreign material is implanted within the heart and vascular system. In this report, we describe and discuss our experience with the implantation of a S-ICD in a patient with AC, who had low QRS voltage and persistent atrial fibrillation precluding successful S-ICD implantation, as well as the challenges encountered during subsequent transvenous lead implantation.
\end{abstract}

\section{Introduction}

The term arrhythmogenic cardiomyopathy (AC) describes a phenotype of progressive fibro-fatty infiltration of the heart muscle, which typically starts in the right ventricle (RV), but may also involve the left ventricle. ${ }^{1}$ The underlying process is generally considered to be genetically determined and several causative mutations have been iden- tified over the recent years, most of them in genes coding for proteins of the intercalated disc. $^{2,3}$ In addition, epigenetic and environmental factors have reported to contribute significantly to disease progression and severity. ${ }^{4-6}$ For example, extensive endurance exercise may enhance adverse remodeling of the ventricular myocardium, predominantly of the $\mathrm{RV}$, through increased workload of the myocardium. ${ }^{5,7}$ The progressive cardiomyocyte loss and infiltration by fibro-fatty tissue translates into an increased risk of developing potentially lethal ventricular arrhythmias and heart failure. In fact, a large proportion of AC patients is only identified after presentation with palpitations, (pre-)syncope or aborted sudden cardiac death (SCD). Besides diagnostic work-up and medical management of heart failure, prevention of SCD is of utmost importance in these patients. In clinical practice, transvenous implantable cardioverter-defibrillators (TV-ICDs) are implanted in the majority of AC patients that are at increased risk of developing lifethreatening arrhythmias. ${ }^{1}$ However, implantation of subcutaneous ICDs (S-ICDs) may also be considered in individual cases, particularly due to the potentially lower risk of device-related complications such as endocarditis, since no foreign material is implanted within the heart or vascular system. In this report, we describe our experience in a patient with $\mathrm{AC}$ and persistent atrial fibrillation, in whom S-ICD implantation was unsuccessful, as well as the challenges of subsequent T-ICD implantation.

\section{Case description}

In this patient, who had been a competitive endurance athlete since adolescence, a diagnosis of definite $\mathrm{AC}$ according to the 2010 Task Force Criteria was made at the age of 52 years. He had no history of syncope or chest pain, but a positive family history for SCD (uncle). His chief complaint at presentation was a recently manifested exercise intolerance after his only complaint for many years was occasional irregular pulse, which improved during exercise.

The diagnosis was established based on ECG (major repolarization), MRI (major criterion) and arrhythmia criteria (major criterion) after he had presented to the hospital for diagnostic work-up of chronic heart failure and atrial fibrillation (AF). ECG changes included $\mathrm{T}$ wave inversions in leads V1 to V4 (major criterion) as well as in III and aVF (Figure 1A). Transthoracic echocardiography and cardiac MRI demonstrated severe right ventricular (RV) dilatation with reduced function ( $\mathrm{RV}$ end-diastolic area $18 \mathrm{~cm}^{2} / \mathrm{m}^{2}$, a fractional area change (FAC) of $22 \%$; Figure 1B), severe tricuspid regurgitation, and severe right atri-
Correspondence: Peter C. Kahr, Department of Cardiology, University Heart Centre Zurich, Rämistrasse 100, 8091 Zurich, Switzerland.

Tel.: +41.44.255.3422 - Fax: +41.44 255.4401

E-mail: pckahr@gmail.com

Key words: Arrhythmogenic right ventricular cardiomyopathy; subcutaneous implantable cardioverter/defibrillator; arrhythmia; sudden cardiac death.

Acknowledgments: the authors would like to thank PD Dr. Robert Manka for providing the MRI image in Figure 1.

Conflict of interest: AMS received a speaker honorarium from Boston Scientific. JS reports personal fees from Amgen, Astra Zeneca, Boehringer-Ingelheim, Bristol-Myers Squibb, Cook Medical, Novartis, Pfizer, SanofiAventis, Sorin, Zoll and Atricure. JS reports grants and personal fees from Bayer Healthcare, Biosense Webster, Boston Scientific, Daiichi-Sankyo, Medtronic, St. Jude Medical/Abbott and Biotronik. JS is owner of CorXL. The other authors report no conflicts of interest. The Zurich ARVC Program is supported by grants from the Bertha and Georg Schwyzer-Winiker Foundation, Baugarten Foundation and the Swiss National Foundation.

Received for publication: 26 August 2017 Accepted for publication: 4 November 2017.

This work is licensed under a Creative Commons Attribution NonCommercial 4.0 License (CC BY-NC 4.0).

CCopyright P.C. Kahr et al., 2017

Licensee PAGEPress, Italy

Cardiogenetics 2017; 7:7025

doi:10.4081/cardiogenetics.2017.7025

al (RA) dilatation (right atrial volume index $49 \mathrm{~mL} / \mathrm{m}^{2}$ ). The RV subtricuspid region and RV free wall appeared thinned and showed disseminated microaneurysms. The RV outflow tract (RVOT) was dyskinetic. At the same time, left ventricular (LV) function was slightly reduced (ejection fraction $46 \%$ ) with moderate mitral regurgitation and severe dilatation of the left atrium (left atrial volume index $\left.66 \mathrm{~mL} / \mathrm{m}^{2}\right)$. Late gadolinium enhancement (LGE) was not observed in the LV. Although difficult to interpret based on the thinned RV wall, the MRI sequences were suggestive of LGE in the RV (arrowheads in Figure 1C). Pathological work-up of RV endomyocardial biopsies demonstrated interstitial fibrosis and partial replacement of cardiomyocytes, underscoring a diagnosis of AC. Genetic testing including 94 cardiomyopathy/channelopathy associated genes with 
next-generation-sequencing did not reveal any pathogenic mutation.

\section{Electrical phenotype}

Upon first presentation to our clinic, the patient presented with AF (which may have been the reason for his chief complaint at presentation) and the typical ECG changes suggestive of AC described above. ${ }^{8}$ At rest, he had sinus bradycardia (min. $34 \mathrm{bpm}$ on $48 \mathrm{~h}$ Holter-ECG), but responded adequately to exercise (max. $177 \mathrm{bpm}$ ). Exercisetesting revealed pleomorphic ventricular premature beats and non-sustained ventricular tachycardia (VT; up to 9 beats) from the inferior RV (left-bundle branch block morphology, superior axis) at maximal intensity and during the early recovery period. Repeated 48h Holter-ECG revealed at least four foci of PVC (PVC burden 4\%) along with a few runs of non-sustained VT. During invasive electrophysiological study, pleomorphic non-sustained VTs, including one with left-bundle branch block morphology and superior axis were induced. Endocardial voltage mapping revealed a bipolar low voltage area/scar (cut-off $<1.5$ $\mathrm{mV}$ ) with fractionated potentials in the subtricuspid region, which was well in line with the echocardiographic and MRI findings. Abnormal unipolar voltages (cut-off $<5.5 \mathrm{mV}$ ) were identified in the subtricuspid region and in the anterior RVOT, implying a larger epicardial substrate as compared to the endocardial one. A voltage map of the right atrium confirmed severe dilatation of this chamber $(450 \mathrm{~mL})$, but bipolar voltages (cut-off $<0.5 \mathrm{mV}$ ) were surprisingly normal, which well corresponds to the large $\mathrm{P}$ waves visible during sinus rhythm and AF on the surface ECG (Figure 1A).

\section{ICD selection and implantation pro- cedure}

Based on the clinical findings and a risk for major adverse cardiac events between 1$10 \%$ per year (intermediate risk non-sustained VT, FAC of $22 \%$ ) according to a recent position paper (class IIa recommendation $^{9}$ ), the decision for primary prophylactic ICD implantation was made. Because there was no documentation and no inducibility of monomorphic sustained VT requiring anti-tachycardia pacing (ATP), no pacemaker dependency (normal chronotropic competence during exercise testing), and no need for cardiac resynchronization, this relatively young patient (52 years at implantation) opted for a S-ICD). Further arguments included the potentially reduced risk of endocarditis with a subcutaneous device, as well as a potentially lower rate of lead failures due to a more robust lead design without an inner lumen of the SICD lead and fewer shear forces as com- pared to a transvenous lead in the beating heart. ${ }^{10}$ Manual screening for eligibility during AF with the Boston Scientific ${ }^{\circledR}$ Programmer revealed at least one out of three ECG vectors to be suitable in all postural positions (Figure 1D, asterisk indicates an acceptable vector).

S-ICD implantation (Boston Scientific ${ }^{\circledR}$ EMBLEM) was performed under general anesthesia in our electrophysiological laboratory under standard sterile conditions. To ensure a correct positioning of the generator and the electrode, a dummy was placed on the skin surface under guidance of anatomi- cal landmarks and the position confirmed via fluoroscopy prior to the actual intervention. The S-ICD implantation was performed following the standard operating procedure ${ }^{10}$ with the device placed between the serratus anterior and latissimus dorsi muscles using a 2-incision technique tunneling the electrode left parasternal from the xiphoid area towards the substernal notch using an $11 \mathrm{~F}$ peel-away sheath. Intraoperative testing of the device during AF only revealed a single vector to be suitable for QRS detection (secondary vector). However, unexpectedly, we observed significant oversensing of atrial
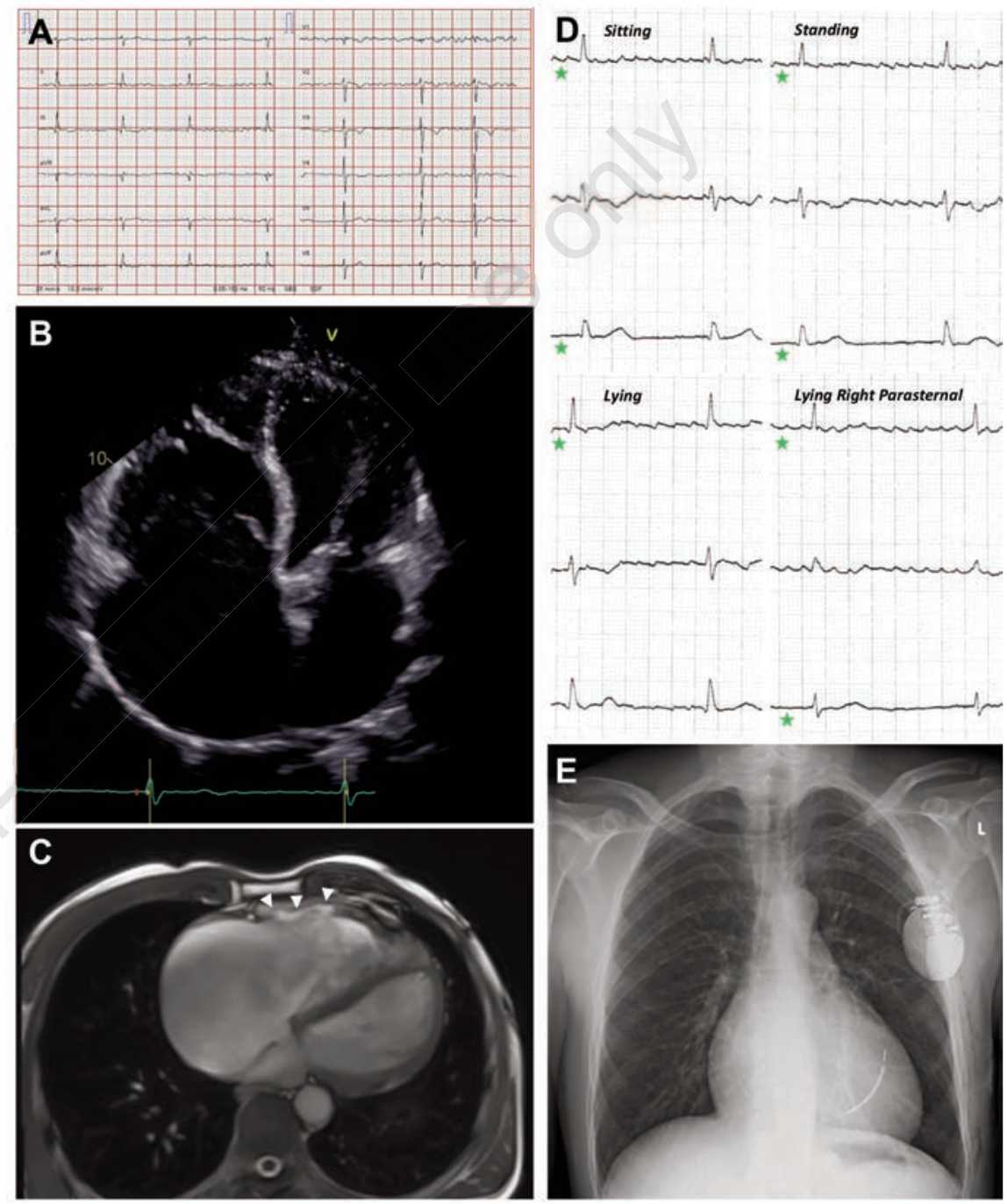

Figure 1. Phenotypical findings in this patient with AC. A) ECG changes typical for rightdominant $\mathrm{AC}$ include negative $\mathrm{T}$-waves in $\mathrm{V} 1$ to $\mathrm{V} 4$ as well as flattened $\mathrm{T}$ waves in the limb leads. The patient presented in atrial fibrillation. B) Transthoracic echocardiography demonstrated a severely dilated RV with wall motion abnormalities, along with severe dilation of the right atrium. Systolic function was reduced in both ventricles. C) MRI found late gadolinium enhancement (arrowheads) in the basal portion of the RV free wall. D) Manual screening for S-ICD implantation, including for a right parasternal position of the device, revealed at least one possible detection vector (green arrows) when the patient was lying, sitting or standing. E) Chest X-ray (a.p.) of the final position of the implanted transvenous device illustrates the position of the single-coil ICD electrode high in the right ventricular septum. 
activation during atrial fibrillation by the device (red box in Figure 2A). In an attempt to test the sensing properties of the S-ICD during tachycardia, since arrhythmias in AC usually occur during exercise, isoproterenol was started. This improved the discrimination between QRS complexes and AF (Figure 2B). Next, a periprocedural device defibrillation test was performed by induction of ventricular fibrillation (VF) using a $50 \mathrm{~Hz}$ burst through the S-ICD. VF was correctly identified by the device (green box in Figure 2C). However, the VF episode terminated spontaneously. At this point, with the S-ICD being more sensitive after previous detection of a shockable rhythm (VF), atrial activity during AF was inappropriately oversensed again, initiating tachycardia detection, and the S-ICD started charging for shock delivery, which was manually aborted. Therefore, due to the high risk of oversensing of atrial activity during AF and misclassification as QRS complexes, due to suboptimal screening of the right parasternal position, and the risk of inappropriate shocks with the S-ICD, we decided to remove the SICD including the lead, and to implant a conventional single-lead single-coil transvenous ICD (Boston Scientific ${ }^{\circledR}$ Autogen). As expected, positioning the RV lead was difficult due to the extensive RV fibro-fatty substrate. Finally, a high septal RV position was achieved with acceptable parameters (Figure 1E).

\section{Discussion}

\section{Indications for ICD Implantation in patients with $\mathrm{AC}$}

Although current guidelines recommend ICD implantation in AC patients after aborted SCD, hemodynamically unstable VT or VF (class I recommendation), primary prevention of SCD in AC patients with a lower risk profile is somewhat controversial. ${ }^{9,12}$ Based on the most current international consensus statement, severe dysfunction of the RV and/or LV are also considered as class I indications for ICD implantation. ${ }^{9}$ ICD implantation should also be considered in patients with an intermediate risk (estimated annual event rate between 1 and $10 \%$ ), when at least one of the following factors is present: hemodynamically stable sustained VT or non-sustained VT, history of syncope or moderate RV and/or LV dysfunction (class IIa). ${ }^{9,11}$ Furthermore, implantation may be considered in patients with minor risk factors including male gender, younger age, certain ECG abnormalities or electrophysiological inducibility of VT/VF during electrophysio- logical studies (class IIb). ${ }^{9,11}$ Implantation should be avoided in asymptomatic patients without risk factors. In conclusion, the decision for ICD implantation in this patient without a history of aborted SCD, sustained VT or history of syncope was based on reduced ventricular function, the documentation of spontaneous non-sustained VT along with the inducibility of non-sustained VT upon electrophysiological investigation, and a family history for SCD (class IIa recommendation $^{9}$ ).
A

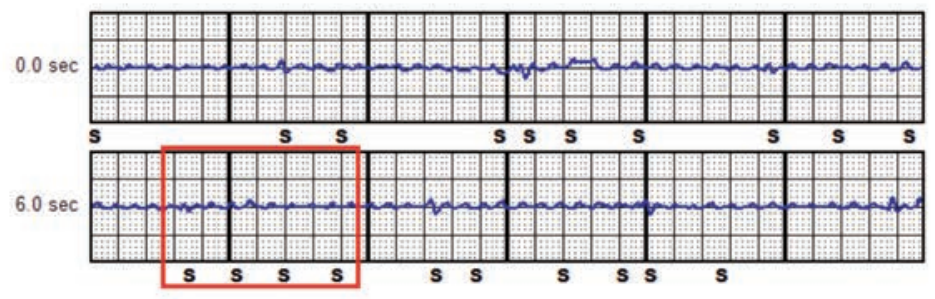

B

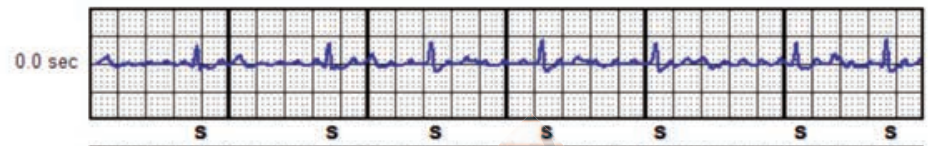

$6.0 \mathrm{sec}$ s s $\quad s$

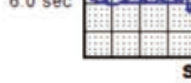

C
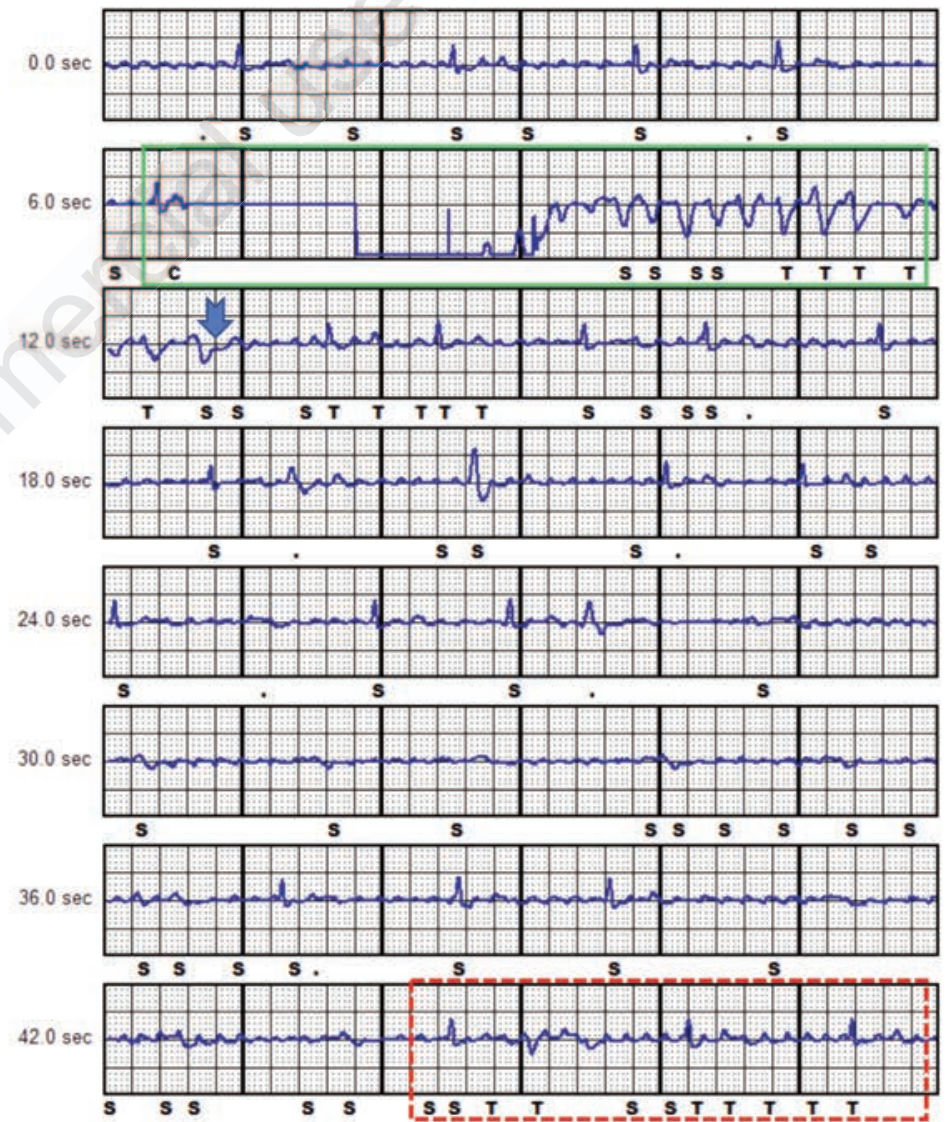

Figure 2. S-ICD tracings from intra-operative testing. A) The S-ICD falsely recognizes atrial fibrillation waves as $Q R S$ complexes ( $S$ = sensing of a $Q R S$ complex, red box). B) After administration of isoproterenol $(3 \mathrm{ug} / \mathrm{min}$.), QRS detection improves, but still is far from perfect. C) Ventricular fibrillation is induced for defibrillation testing. VF (T) is detected with some QRS drop outs (green box) and then terminates spontaneously (blue arrow). Finally, the device senses atrial fibrillation waves again as QRS complexes and therefore as ventricular tachyarrhythmia $(T)$ due to an increased sensitivity after a previous episode of VF) (red dotted box), and the device starts charging the defibrillator, which was manually aborted (not shown). 
Arguments favoring implantation of a S-ICD in patients with $A C$

The general advantages of S-ICDs over traditional TV-ICDs also apply to patients with AC: morbidity is lower due to the absence of life-threatening complications such as pericardial tamponade or haemato/pneumothorax during the implantation procedure. Device-related complications over the long-term are probably higher in TVICDs as they are mainly lead-related. The S-ICD lead has a different structure as compared to TV-ICD leads with no central lumen resulting in a greater tensile strength and higher robustness. Additionally, the subcutaneous as opposed to intracardiac localization exposes the lead to less physical stress. Recent observational data have confirmed the low risk of lead malfunctions with only $0.8 \%$ over 5 years (as compared to $15 \%$ in the matched TV-ICD cohort). With regard to this, the necessity of lead extraction is probably lower with the S-ICD system, and the extraction procedure itself by far less invasive than that of transvenous leads. ${ }^{12,13}$ The complete extravascular localization makes the system less prone to systemic infection and eliminates the risk of device-related endocarditis. ${ }^{14}$ Indeed, the risk for device-associated infection in the pooled EFFORTLESS and IDE registries was low with $1.7 \% .{ }^{15}$ Of note, all infections were local and no case of endovascular infection has been documented so far.

In our case, the decision to implant a subcutaneous system was mainly based on these arguments. Additionally, positioning of the ICD electrode within the dilated and dysplastic RV of AC patients may be difficult (as then observed in the subsequent TVICD implantation in the same patient). On the downside, the advantages of transvenous devices over S-ICDs may be required in patients with $\mathrm{AC}$, i.e. the ability of antitachycardia pacing (ATP) as well as antibradycardia pacing. In this patient, we concluded that ATP was unlikely because no spontaneous sustained VT had been documented so far, and no sustained VT could be induced during electrophysiological testing. Despite a rather low heart rate at rest, Holter ECG and stress testing documented adequate increases in heart rate upon physical exercise in this athletic patient. Therefore, we concluded that antibradycardia pacing was also very unlikely to be required.

\section{Arguments against the implantation of an S-ICD in patients with AC}

As illustrated by this case, progressive right and left ventricular loss of cardiomyocytes in AC results in reduced voltages of the surface QRS complexes. This may significantly hamper arrhythmia detection by the S-ICD, since its arrhythmia detection algorithm is based on the analysis of the surface QRS complexes. At the same time, the dilation of the atria enhances the risk of $\mathrm{AF}$. In this patient, the right atrium was severely enlarged, but bipolar voltages were normal, which well corresponds to the large $\mathrm{P}$ waves visible during sinus rhythm and atrial activity during AF on the surface ECG (Figure 1A). These circumstances increased the difficulty for the device to discriminate between atrial and ventricular complexes. As illustrated by this case, misclassification of AF waves as QRS complexes can lead to an increased risk of inappropriate shocks. This electroanatomical feature of patients with $\mathrm{AC}$, which can be aggravated as the disease progresses over years, may be a main argument against S-ICDs in AC.

\section{Conclusions}

Implantation of ICDs is commonly performed in patients with $\mathrm{AC}$ that are at an increased risk of SCD. Although implantation of a S-ICD may be a viable option due to a lower risk of endocarditis and lead malfunction in this relatively young patient population, the usefulness of S-ICDs may be limited by the electroanatomical features of this progressive disease - low voltages of the QRS complexes and increased risk of $\mathrm{AF}$ - predisposing these individuals to an increased risk of inappropriate shocks.

\section{References}

1. Saguner AM, Brunckhorst C, Duru F. Arrhythmogenic ventricular cardiomyopathy: a paradigm shift from right to biventricular disease. World J Cardiol 2014;6:154-74.

2. Fressart V, Duthoit G, Donal E, et al. Desmosomal gene analysis in arrhythmogenic right ventricular dysplasia/cardiomyopathy: spectrum of mutations and clinical impact in practice. Europace 2010;12:861-8.

3. Saguner AM, Duru F, Brunckhorst CB. Arrhythmogenic right ventricular cardiomyopathy: a challenging disease of the intercalated disc. Circulation 2013;128:1381-6.

4. Pinamonti B, Dragos AM, Pyxaras SA, et al. Prognostic predictors in arrhythmogenic right ventricular cardiomyopathy: results from a 10-year registry. Eur Heart J 2011;32:1105-13.

5. James CA, Bhonsale A, Tichnell C, et al. Exercise increases age-related penetrance and arrhythmic risk in arrhythmogenic right ventricular dysplasia/car- diomyopathy-associated desmosomal mutation carriers. J Am Coll Cardiol 2013;62:1290-7.

6. Akdis D, Saguner AM, Shah K, et al. Sex hormones affect outcome in arrhythmogenic right ventricular cardiomyopathy/dysplasia: from a stem cell derived cardiomyocyte-based model to clinical biomarkers of disease outcome. Eur Heart J 2017;38:1498508.

7. Saberniak J, et al. Vigorous physical activity impairs myocardial function in patients with arrhythmogenic right ventricular cardiomyopathy and in mutation positive family members. Eur J Heart Fail 2014;16:1337-44.

8. Saguner AM, Hasselberg NE, Borgquist $\mathrm{R}$, et al. Electrocardiographic features of disease progression in arrhythmogenic right ventricular cardiomyopathy/dysplasia. BMC Cardiovasc Disord 2015;15:4.

9. Corrado D, Wichter T, Link MS, et al. Treatment of arrhythmogenic right ventricular cardiomyopathy/dysplasia: an international task force consensus statement. Eur Heart J 20153;6:3227-37.

10. Ozkartal T, Breitenstein A, Saguner AM, et al. The subcutaneous implantable cardioverter defibrillator in daily clinical practice. Swiss Med Wkly 2017;147:w14518.

11. Priori SG, Blomström-Lundqvist C, Mazzanti A, et al. 2015 ESC Guidelines for the management of patients with ventricular arrhythmias and the prevention of sudden cardiac death: The Task Force for the Management of Patients with Ventricular Arrhythmias and the Prevention of Sudden Cardiac Death of the European Society of Cardiology (ESC). Endorsed by: Association for European Paediatric and Congenital Cardiology (AEPC). Eur Heart J 2015;36:2793-867.

12. Maytin M, Jones SO, Epstein L. M. Long-term mortality after transvenous lead extraction. Circulation 2012;5:2527.

13. Maytin M, Epstein LM. The challenges of transvenous lead extraction. Heart 2011;97:425-34.

14. Lewis GF, Gold MR. Safety and efficacy of the subcutaneous implantable defibrillator. J Am Coll Cardiol 2016; 67:445-54.

15. Burke MC, Gold MR, Knight BP, et al. Safety and efficacy of the totally subcutaneous implantable defibrillator: 2year results from a pooled analysis of the IDE study and EFFORTLESS Registry. J Am Coll Cardiol 2015;65: 1605-15. 